\title{
MIMO-OTFS in High-Doppler Fading Channels: Signal Detection and Channel Estimation
}

\author{
M. Kollengode Ramachandran and A. Chockalingam \\ Department of ECE, Indian Institute of Science, Bangalore 560012
}

\begin{abstract}
Orthogonal time frequency space (OTFS) modulation is a recently introduced multiplexing technique designed in the 2-dimensional (2D) delay-Doppler domain suited for highDoppler fading channels. OTFS converts a doubly-dispersive channel into an almost non-fading channel in the delay-Doppler domain through a series of $2 \mathrm{D}$ transformations. In this paper, we focus on MIMO-OTFS which brings in the high spectral and energy efficiency benefits of MIMO and the robustness of OTFS in high-Doppler fading channels. The OTFS channelsymbol coupling and the sparse delay-Doppler channel impulse response enable efficient MIMO channel estimation in high Doppler environments. We present an iterative algorithm for signal detection based on message passing and a channel estimation scheme in the delay-Doppler domain suited for MIMO-OTFS. The proposed channel estimation scheme uses impulses in the delay-Doppler domain as pilots for estimation. We also compare the performance of MIMO-OTFS with that of MIMO-OFDM under high Doppler scenarios.
\end{abstract}

keywords: OTFS modulation, MIMO-OTFS, $2 D$ modulation, delayDoppler domain, MIMO-OTFS signal detection, channel estimation.

\section{INTRODUCTION}

Future wireless systems including $5 \mathrm{G}$ systems need to operate in dynamic channel conditions, where operation in high mobility scenarios (e.g., high-speed trains) and millimeter wave (mm Wave) bands are envisioned. The wireless channels in such scenarios are doubly-dispersive, where multipath propagation effects cause time dispersion and Doppler shifts cause frequency dispersion [1]. OFDM systems are usually employed to mitigate the effect of inter-symbol interference (ISI) caused by time dispersion [2]. However, Doppler shifts result in inter-carrier interference (ICI) in OFDM and degrades performance [3]. An approach to jointly combat ISI and ICI is to use pulse shaped OFDM systems [4]-[6]. Pulse shaped OFDM systems use general time-frequency lattices and optimized pulse shapes in the time-frequency domain. However, systems that employ the pulse shaping approach do not efficiently address the need to support high Doppler shifts.

Orthogonal time frequency space (OTFS) modulation is a recently proposed multiplexing scheme [7]-[10] which meets the high-Doppler signaling need through a different approach, namely, multiplexing the modulation symbols in the delayDoppler domain (instead of multiplexing symbols in timefrequency domain as in traditional modulation techniques such as OFDM). OTFS waveform has been shown to be resilient to delay-Doppler shifts in the wireless channel. For example, OTFS has been shown to achieve significantly better error

This work was supported in part by the J. C. Bose National Fellowship, Department of Science and Technology, Government of India. performance compared to OFDM for vehicle speeds ranging from $30 \mathrm{~km} / \mathrm{h}$ to $500 \mathrm{~km} / \mathrm{h}$ in $4 \mathrm{GHz}$ band, and that the robustness to high-Doppler channels (e.g., $500 \mathrm{~km} / \mathrm{h}$ vehicle speeds) is especially notable, as OFDM performance breaks down in such high-Doppler scenarios [9]. When OTFS waveform is viewed in the delay-Doppler domain, it corresponds to a 2D localized pulse. Modulation symbols, such as QAM symbols, are multiplexed using these pulses as basis functions. The idea is to transform the time-varying multipath channel into a 2D time-invariant channel in the delay-Doppler domain. This results in a simple and symmetric coupling between the channel and the modulation symbols, due to which significant performance gains compared to other multiplexing techniques are achieved [7]. OTFS modulation can be architected over any multicarrier modulation by adding pre-processing and postprocessing blocks. This is very attractive from an implementation view-point.

Recognizing the promise of OTFS in future wireless systems, including mmWave communication systems [10], several works on OTFS have started emerging in the recent literature [11]-[17]. These works have addressed the formulation of input-output relation in vectorized form, equalization and detection, and channel estimation. Multiple-input multiple-output (MIMO) techniques along with OTFS (MIMO-OTFS) can achieve increased spectral/energy efficiencies and robustness in rapidly varying MIMO channels. It is shown in [7] that OTFS approaches channel capacity through linear scaling of spectral efficiency with the MIMO order. We, in this paper, consider the signal detection and channel estimation aspects in MIMO-OTFS.

Our contributions can be summarized as follows. We first present a vectorized input-output formulation for the MIMOOTFS system. This linear vector channel model enables MIMO-OTFS signal detection using a variety of detection algorithms. Initially, we assume perfect channel knowledge at the receiver and employ an iterative algorithm based on message passing for signal detection. The algorithm has low complexity and it achieves very good performance. For example, in a $2 \times 2$ MIMO-OTFS system, a bit error rate (BER) of $10^{-5}$ is achieved at an SNR of about $14 \mathrm{~dB}$ for a Doppler of $1880 \mathrm{~Hz}(500 \mathrm{~km} / \mathrm{hr}$ speed at $4 \mathrm{GHz})$. For the same system, MIMO-OFDM BER performance floors at a BER of 0.02. Next, we relax the perfect channel estimation assumption and present a channel estimation scheme in the delay-Doppler domain. The proposed scheme uses impulses in the delay-Doppler domain as pilots for MIMO-OTFS channel estimation. The proposed scheme is simple and effective in 


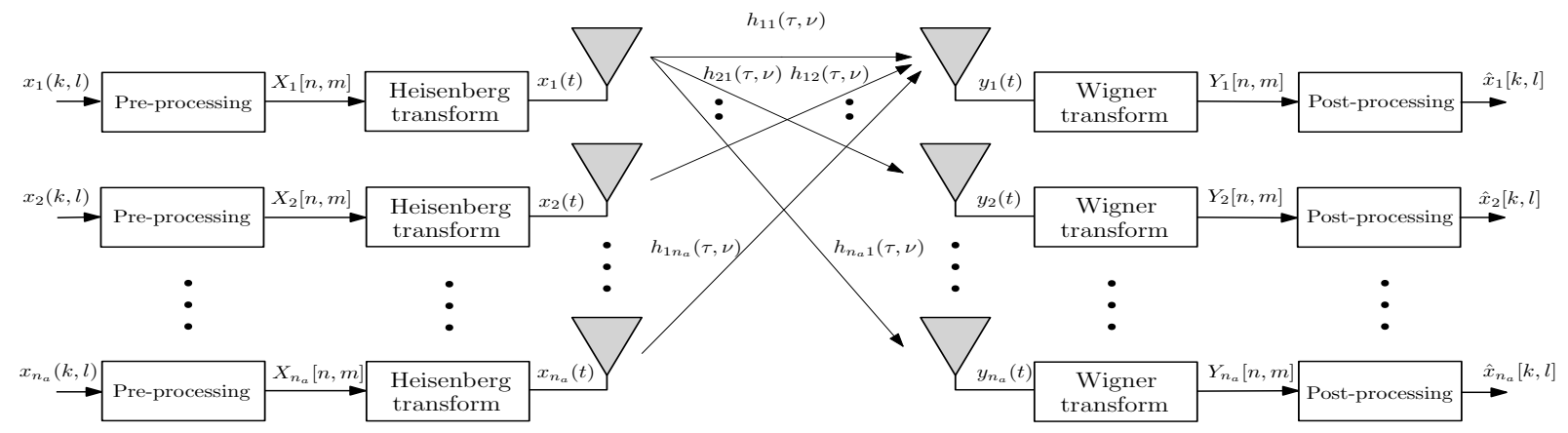

Fig. 1. MIMO-OTFS modulation scheme.

high-Doppler MIMO channels. For example, compared to the case of perfect channel knowledge, the proposed scheme loses performance only by less than a fraction of a $\mathrm{dB}$.

The rest of the paper is organized as follows. The MIMOOTFS system model and the vectorized input-output relation are developed in Sec. II. MIMO-OTFS signal detection using message passing and the resulting BER performance are presented in Sec. III. The channel estimation scheme in the delayDoppler domain and the achieved performance are presented in Sec. IV. The conclusions are presented in Sec. V.

\section{MIMO-OTFS MODULATION}

Consider a MIMO-OTFS system as shown in Fig. 1 with equal number of transmit $\left(n_{t}\right)$ and receive antennas $\left(n_{r}\right)$, i.e., $n_{t}=n_{r}=n_{a}$. The treatment can be extended to the case of $n_{r} \geq n_{t}$. Please refer [12],[13] for the system model of a SISO-OTFS system and the development of a vectorized formulation of the input-output relation in SISOOTFS. Each antenna in the MIMO-OTFS system transmits OTFS modulated information symbols independently. Let the windows $W_{t x}[n, m], W_{r x}[n, m]$ used for modulation be rectangular. Assume that the channel corresponding to $p$ th transmit antenna and $q$ th receive antenna has $P$ taps. So the channel representation can be written as

$$
h_{q p}(\tau, \nu)=\sum_{i=1}^{P} h_{q p_{i}} \delta\left(\tau-\tau_{i}\right) \delta\left(\nu-\nu_{i}\right),
$$

where $\tau_{i}, \nu_{i}$, and $h_{q p_{i}}$ denote the delay, Doppler, and fade coefficient of the $i$ th path, respectively, and $p=1,2, \cdots, n_{a}$, $q=1,2, \cdots, n_{a}$. We can use the vectorized formulation in [12],[13] for each transmit and receive antenna pair to describe the input-output relation.

\section{A. Vectorized formulation of the input-output relation for MIMO-OTFS}

Let $\mathbf{H}_{q p}$ denote the equivalent channel matrix corresponding to $p$ th transmit antenna and $q$ th receive antenna. Let $\mathbf{x}_{p}$ denote the $N M \times 1$ transmit vector from the $p$ th transmit antenna and $\mathbf{y}_{q}$ denote the $N M \times 1$ received vector corresponding to $q$ th receive antenna in a given frame. Similar to the system model in [12],[13] for a SISO-OTFS system, we can derive a linear system model describing the input and output for the MIMOOTFS system as given below

$$
\begin{aligned}
& \mathbf{y}_{1}=\mathbf{H}_{11} \mathbf{x}_{1}+\mathbf{H}_{12} \mathbf{x}_{2}+\cdots+\mathbf{H}_{1 n_{a}} \mathbf{x}_{n_{a}}+\mathbf{v}_{1}, \\
& \mathbf{y}_{2}=\mathbf{H}_{21} \mathbf{x}_{1}+\mathbf{H}_{22} \mathbf{x}_{2}+\cdots+\mathbf{H}_{2 n_{a}} \mathbf{x}_{n_{a}}+\mathbf{v}_{2} \text {, } \\
& \mathbf{y}_{n_{a}}=\mathbf{H}_{n_{a} 1} \mathbf{x}_{1}+\mathbf{H}_{n_{a} 2} \mathbf{x}_{2}+\cdots+\mathbf{H}_{n_{a} n_{a}} \mathbf{x}_{n_{a}}+\mathbf{v}_{n_{a}} . \\
& \mathbf{H}_{\text {мІмо }}=\left[\begin{array}{cccc}
\mathbf{H}_{11} & \mathbf{H}_{12} & \ldots & \mathbf{H}_{1 n_{a}} \\
\mathbf{H}_{21} & \mathbf{H}_{22} & \ldots & \mathbf{H}_{2 n_{a}} \\
\vdots & \vdots & \ddots & \vdots \\
\mathbf{H}_{n_{a} 1} & \mathbf{H}_{n_{a} 2} & \ldots & \mathbf{H}_{n_{a} n_{a}}
\end{array}\right],
\end{aligned}
$$

Then, (2) can be written as

$$
\mathbf{y}_{\text {MIMO }}=\mathbf{H}_{\text {MIMO }} \mathbf{x}_{\text {MIMO }}+\mathbf{v}_{\text {MIMO }} \text {, }
$$

where $\quad \mathbf{x}_{\text {мімо }}, \mathbf{y}_{\text {мімо }}, \mathbf{v}_{\text {мімо }} \in \mathbb{C}^{n_{a} N M \times 1}, \mathbf{H}_{\text {мімо }} \in$ $\mathbb{C}^{n_{a} N M \times n_{a} N M}$. Thus, in this representation, each row and column of $\mathbf{H}_{\text {мімо }}$ has only $n_{a} P$ non-zero elements due to modulo operations.

\section{MIMO-OTFS SignAl DETECTION}

In this section, we present a MIMO-OTFS signal detection scheme using an iterative algorithm based on message passing and present a performance comparison between MIMO-OTFS and MIMO-OFDM in high-Doppler scenarios.

\section{A. Algorithm for MIMO-OTFS signal detection}

Let the sets of non-zero positions in the $b$ th row and $a$ th column of $\mathbf{H}_{\text {мімо }}$ be denoted by $\zeta_{b}$ and $\zeta_{a}$, respectively. Using (3), the system can be modeled as a sparsely connected factor graph with $n_{a} N M$ variable nodes corresponding to the elements in $\mathbf{x}_{\text {MIмо }}$ and $n_{a} N M$ observation nodes corresponding to the elements in $\mathbf{y}_{\text {мімо }}$. Each observation node $y_{b}$ is connected to the set of variable nodes $\left\{x_{c}, c \in \zeta_{b}\right\}$, and each variable node $x_{a}$ is connected to the set of observation nodes $\left\{y_{c}, c \in \zeta_{a}\right\}$. Also, $\left|\zeta_{b}\right|=\left|\zeta_{a}\right|=n_{a} P$. The maximum a posteriori (MAP) decision rule for (3) is given by

$$
\hat{\mathbf{x}}_{\text {Мाмо }}=\underset{\mathbf{x}_{\text {MIMо }} \in \mathbb{A}^{n_{a} N M}}{\operatorname{argmax}} \operatorname{Pr}\left(\mathbf{x}_{\text {мІмо }} \mid \mathbf{y}_{\text {мімо }}, \mathbf{H}_{\text {Мाмо }}\right),
$$


where $\mathbb{A}$ is the modulation alphabet (e.g., QAM) used. The detection as per (4) has exponential complexity. Hence, we use symbol by symbol MAP rule for $0 \leq a \leq n_{a} N M-1$ for detection as follows:

$$
\begin{aligned}
\hat{x}_{a} & =\underset{a_{j} \in \mathbb{A}}{\operatorname{argmax}} \operatorname{Pr}\left(x_{a}=a_{j} \mid \mathbf{y}_{\text {мІмо }}, \mathbf{H}_{\mathrm{MIMO}}\right) \\
& =\underset{a_{j} \in \mathbb{A}}{\operatorname{argmax}} \frac{1}{|\mathbb{A}|} \operatorname{Pr}\left(\mathbf{y}_{\mathrm{MIMO}} \mid x_{a}=a_{j}, \mathbf{H}_{\mathrm{MIMO}}\right) \\
& \approx \underset{a_{j} \in \mathbb{A}}{\operatorname{argmax}} \prod_{c \in \zeta_{a}} \operatorname{Pr}\left(y_{c} \mid x_{a}=a_{j}, \mathbf{H}_{\mathrm{MIMO}}\right) .
\end{aligned}
$$

The transmitted symbols are assumed to be equally likely and

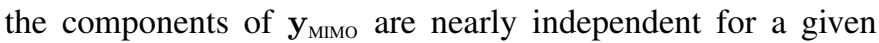
$x_{a}$ due to the sparsity in $\mathbf{H}_{\text {MIмо }}$. This can be solved using the message passing based algorithm described below. The message that is passed from the variable node $x_{a}$, for each $a=\left\{0,1, \cdots, n_{a} N M-1\right\}$, to the observation node $y_{b}$ for $b \in \zeta_{a}$, is the pmf denoted by $\mathbf{p}_{a b}=\left\{p_{a b}\left(a_{j}\right) \mid a_{j} \in \mathbb{A}\right\}$ of the symbols in the constellation $\mathbb{A}$. Let $H_{a b}$ denote the element in the $a$ th row and $b$ th column of $\mathbf{H}_{\text {мIмо }}$. The message passing algorithm is described as follows.

1: Inputs: $\mathbf{y}_{\text {мімо }}, \mathbf{H}_{\text {мімо }}, N_{\text {iter }}$ : max. number of iterations.

2: Initialization: Iteration index $t=0, \operatorname{pmf} \mathbf{p}_{a b}^{(0)}=$ $1 /|\mathbb{A}| \forall a \in\left\{0,1, \cdots, n_{a} N M-1\right\}$ and $b \in \zeta_{a}$.

3: Messages from $y_{b}$ to $x_{a}$ : The mean $\left(\mu_{b a}^{(t)}\right)$ and variance $\left(\left(\sigma_{b a}^{(t)}\right)^{2}\right)$ of the interference term $I_{b a}$ are passed as messages from $y_{b}$ to $x_{a}$. $I_{b a}$ can be approximated as a Gaussian random variable and is given by

$$
I_{b a}=\sum_{c \in \zeta_{b}, c \neq a} x_{c} H_{b, c}+v_{b} .
$$

The mean and variance of $I_{b a}$ are given by

$$
\begin{gathered}
\mu_{b a}^{(t)}=\mathbb{E}\left[I_{b a}\right]=\sum_{c \in \zeta_{b}, c \neq a} \sum_{j=1}^{|\mathbb{A}|} p_{c b}^{(t)}\left(a_{j}\right) a_{j} H_{b, c}, \\
\left(\sigma_{b a}^{(t)}\right)^{2}=\operatorname{Var}\left[I_{b a}\right] \\
=\sum_{\substack{c \in \zeta_{b} \\
c \neq a}}\left(\sum_{j=1}^{|\mathbb{A}|} p_{c b}^{(t)}\left(a_{j}\right)\left|a_{j}\right|^{2}\left|H_{b, c}\right|^{2}-\left|\sum_{j=1}^{|\mathbb{A}|} p_{c b}^{(t)}\left(a_{j}\right) a_{j} H_{b, c}\right|^{2}\right) \\
+\sigma^{2} .
\end{gathered}
$$

4: Messages from $x_{a}$ to $y_{b}$ : Messages passed from variable nodes $x_{a}$ to observation nodes $y_{b}$ is the pmf vector $\mathbf{p}_{a b}^{(t+1)}$ with the elements given by

$$
p_{a b}^{(t+1)}=\Delta p_{a b}^{(t)}\left(a_{j}\right)+(1-\Delta) p_{a b}^{(t-1)}\left(a_{j}\right),
$$

where $\Delta \in(0,1]$ is the damping factor for improving convergence rate, and

$$
p_{a b}^{(t)} \propto \prod_{c \in \zeta_{a}, c \neq b} \operatorname{Pr}\left(y_{c} \mid x_{a}=a_{j}, \mathbf{H}_{\text {мІмо }}\right),
$$

where

$$
\operatorname{Pr}\left(y_{c} \mid x_{a}=a_{j}, \mathbf{H}_{\mathrm{MIMO}}\right) \propto \exp \left(\frac{-\left|y_{c}-\mu_{c a}^{(t)}-H_{c, a} a_{j}\right|^{2}}{\sigma_{c, a}^{2(t)}}\right) .
$$

5: Stopping criterion: Repeat steps 3 \& 4 till $\max _{a, b, a_{j}}\left|p_{a b}^{(t+1)}\left(a_{j}\right)-p_{a b}^{(t)}\left(a_{j}\right)\right|<\epsilon$ (where $\epsilon$ is a small value) or the maximum number of iterations, $N_{i t e r}$, is reached.

6: Output: Output the detected symbol as

$$
\hat{x}_{a}=\underset{a_{j} \in \mathbb{A}}{\operatorname{argmax}} p_{a}\left(a_{j}\right), a \in 0,1,2, \cdots, n_{a} N M-1,
$$

where

$$
p_{a}\left(a_{j}\right)=\prod_{c \in \zeta_{a}} \operatorname{Pr}\left(y_{c} \mid x_{a}=a_{j}, \mathbf{H}_{\mathrm{MIMO}}\right)
$$

\section{B. Vectorized formulation of the input-output relation for MIMO-OFDM}

In this subsection, in order to provide a performance comparison between MIMO-OTFS and MIMO-OFDM, we present the vectorized formulation of the input-output relation for MIMO-OFDM. OFDM uses the TF domain for signaling and channel representation. We will first derive the vectorized formulation for a SISO-OFDM and extend it to MIMOOFDM. For a fair comparison with the OTFS modulation, we will consider $N$ consecutive OFDM blocks (each of size $M$ ) to be one frame, i.e., the transmit vector $\mathbf{x}_{\text {OFDM }} \in \mathbb{C}^{N M \times 1}$, and message passing detection is done jointly over one $N M \times 1$ frame. The time-delay representation $h(\tau, t)$ is related to the delay-Doppler representation $h(\tau, \nu)$ by a Fourier transform along the time axis, and is given by

$$
h(\tau, t)=\sum_{i=1}^{P} h_{i} e^{j 2 \pi \nu_{i} t} \delta\left(\tau-\tau_{i}\right)
$$

Sample the time axis at $t=n T s=\frac{n}{M \Delta f}$. The sampled timedelay representation $h(\tau, n)$ is given by

$$
h(\tau, n)=\sum_{i=1}^{P} h_{i} e^{\frac{j 2 \pi \nu_{i} n}{M \Delta f}} \delta\left(\tau-\tau_{i}\right) .
$$

Let $C P=P-1$ denote the cyclic prefix length used in each OFDM block and let $L=M+C P$. The size of one frame after cyclic prefix insertion to each block will then be $N L$. Let $\mathbf{T}_{C P}=\left[\begin{array}{ll}\mathbf{C}_{C P}^{T} & \mathbf{I}_{M}\end{array}\right]^{T}$ denote the $L \times M$ matrix that inserts cyclic prefix for one block, where $\mathbf{C}_{C P}$ contains the last $C P$ rows of the identity matrix $\mathbf{I}_{M}$. Also, let $\mathbf{R}_{C P}=\left[\mathbf{0}_{M \times C P} \mathbf{I}_{M}\right]$ denote the $M \times L$ the matrix that removes the cyclic prefix for one block [19]. Let $\mathbf{W}_{M \times M}$ and $\mathbf{W}_{M \times M}^{H}$ denote the DFT and IDFT matrices of size $M$. We use the following notations.

- $\mathbf{B}_{\text {cpin }}=\operatorname{diag} \underbrace{\left(\mathbf{T}_{C P}, \mathbf{T}_{C P}, \cdots, \mathbf{T}_{C P}\right)}_{N \text { times }}:$ cyclic prefix insertion matrix for $N$ consecutive OFDM blocks.

- $\mathbf{B}_{\text {cpre }}=\operatorname{diag} \underbrace{\left(\mathbf{R}_{C P}, \mathbf{R}_{C P}, \cdots, \mathbf{R}_{C P}\right)}_{N \text { times }}:$ cyclic prefix removal matrix for $N$ consecutive OFDM blocks.

- $\mathbf{D}=\operatorname{diag} \underbrace{(\mathbf{W}, \mathbf{W}, \cdots, \mathbf{W})}_{N \text { times }}:$ DFT matrix for $N$ consecutive OFDM blocks. 
- $\mathbf{D}^{H}=\operatorname{diag} \underbrace{\left(\mathbf{W}^{H}, \mathbf{W}^{H}, \cdots, \mathbf{W}^{H}\right)}_{\text {Ntimes }}$ : IDFT matrix for $N$ consecutive OFDM blocks.

- The channel in the time-delay domain for a given frame can be written as a matrix $\mathbf{H}_{t d}$ using (11) and has size $N L \times N L$.

Using the above, the end-to-end relationship in OFDM modulation can be described by the following linear model:

$$
\begin{aligned}
\mathbf{y}_{\text {OFDM }} & =\underbrace{\mathbf{D B}_{c p r e} \mathbf{H}_{t d} \mathbf{B}_{c p i n} \mathbf{D}^{H}}_{\mathbf{H}_{\text {OFDM }}} \mathbf{x}_{\text {OFDM }}+\mathbf{v} \\
& =\mathbf{H}_{\text {OFDM }} \mathbf{x}_{\text {OFDM }}+\mathbf{v},
\end{aligned}
$$

where $\mathbf{x}_{\text {OFDM }}, \mathbf{y}_{\text {OFDM }}, \mathbf{v} \in \mathbb{C}^{N M \times 1}, \mathbf{H}_{\text {OFDM }} \in \mathbb{C}^{N M \times N M}$.

1) MIMO-OFDM: The vectorized formulation of the inputoutput relation for SISO-OFDM derived above can be extended to MIMO-OFDM in a similar fashion as was done for the MIMO-OTFS system described in Sec. II-A . Let $\mathbf{H}_{\text {OFDM }}{ }_{q p}$ denote the equivalent channel matrix corresponding to $p$ th transmit antenna and $q$ th receive antenna. Let $\mathbf{x}_{\mathrm{OFDM}_{p}}$ denote the $N M \times 1$ transmit vector from the $p$ th transmit antenna and $\mathbf{y}_{\text {OFDM }_{q}}$ denote the $N M \times 1$ received vector corresponding to $q$ th receive antenna in a given frame. Define

$$
\begin{aligned}
& \mathbf{H}_{\mathrm{MIMO}-\mathrm{OFDM}}=\left[\begin{array}{cccc}
\mathbf{H}_{\mathrm{OFDM}_{11}} & \mathbf{H}_{\mathrm{OFDM}_{12}} & \cdots & \mathbf{H}_{\mathrm{OFDM}_{1 n_{a}}} \\
\mathbf{H}_{\mathrm{OFDM}_{21}} & \mathbf{H}_{\mathrm{OFDM}_{22}} & \cdots & \mathbf{H}_{\mathrm{OFDM}_{2 n}} \\
\vdots & \vdots & \ddots & \vdots \\
\mathbf{H}_{\mathrm{OFDM}_{n_{a} 1}} & \mathbf{H}_{\mathrm{OFDM}_{n_{a} 2}} & \cdots & \mathbf{H}_{\mathrm{OFDM}_{n_{a} n_{a}}}
\end{array}\right], \\
& \mathbf{x}_{\text {MIMO-OFDM }}=\left[\mathbf{x}_{\mathrm{OFDM}_{1}}{ }^{T}, \mathbf{x}_{\mathrm{OFDM}_{2}}{ }^{T}, \cdots, \mathbf{x}_{\mathrm{OFDM}_{n a}}{ }^{T}\right]^{T} \text {, } \\
& \mathbf{y}_{\text {MIMO-OFDM }}=\left[\mathbf{y}_{\mathrm{OFDM}_{1}}{ }^{T}, \mathbf{y}_{\mathrm{OFDM}_{2}}{ }^{T}, \cdots, \mathbf{y}_{\mathrm{OFDM}_{n a}}{ }^{T}\right]^{T} \text {. }
\end{aligned}
$$

The input-output relation for MIMO-OFDM can be written as

$$
\mathbf{y}_{\text {MIMO-OFdM }}=\mathbf{H}_{\text {MIMO-OFDM }} \mathbf{x}_{\text {MIMO-OFDM }}+\mathbf{v}_{\text {MIMO-OFDM }},
$$

where $\quad \mathbf{x}_{\text {MIMo-ofdm }}, \mathbf{y}_{\text {Mimo-ofdm }}, \mathbf{v}_{\text {Mimo-ofdm }} \in \mathbb{C}^{n_{a} N M \times 1}$, $\mathbf{H}_{\text {Mimo-ofdm }} \in \mathbb{C}^{n_{a} N M \times n_{a} N M}$.

\section{Performance results and discussions}

In this subsection, we present the BER performance of MIMO-OTFS and compare it with that of MIMO-OFDM. Perfect channel knowledge is assumed at the receiver. Message passing algorithm is used for both MIMO-OTFS and MIMOOFDM. A damping factor of 0.5 is used. The maximum number of iterations and the $\epsilon$ value used are 30 and 0.01 , respectively. We use the channel model in (1) and the number of taps $P$ is taken to be 5. The delay-Doppler profile considered in the simulation is shown in Table I. Other simulation parameters used are given in Table II.

\begin{tabular}{|c|c|c|c|c|c|}
\hline Path index $(i)$ & 1 & 2 & 3 & 4 & 5 \\
\hline Delay $\left(\tau_{i}\right), \mu \mathrm{s}$ & 2.08 & 4.164 & 6.246 & 8.328 & 10.41 \\
\hline Doppler $\left(\nu_{i}\right), \mathrm{Hz}$ & 0 & 470 & 940 & 1410 & 1880 \\
\hline
\end{tabular}

TABLE I

DELAY-DOPPLER PROFILE FOR THE CHANNEL MODEL WITH $P=5$.

Figure 2 shows the BER performance of MIMO-OTFS for SISO as well as $2 \times 2$ and $3 \times 3$ MIMO configurations.

\begin{tabular}{|l|l|}
\hline Parameter & Value \\
\hline Carrier frequency $(\mathrm{GHz})$ & 4 \\
\hline Subcarrier spacing $(\mathrm{kHz})$ & 15 \\
\hline Frame size $(M, N)$ & $(32,32)$ \\
\hline Modulation scheme & BPSK \\
\hline MIMO configuration & $1 \times 1,2 \times 2,3 \times 3$ \\
\hline Maximum speed $(\mathrm{kmph})$ & 507.6 \\
\hline
\end{tabular}

TABLE II

SYSTEM PARAMETERS.

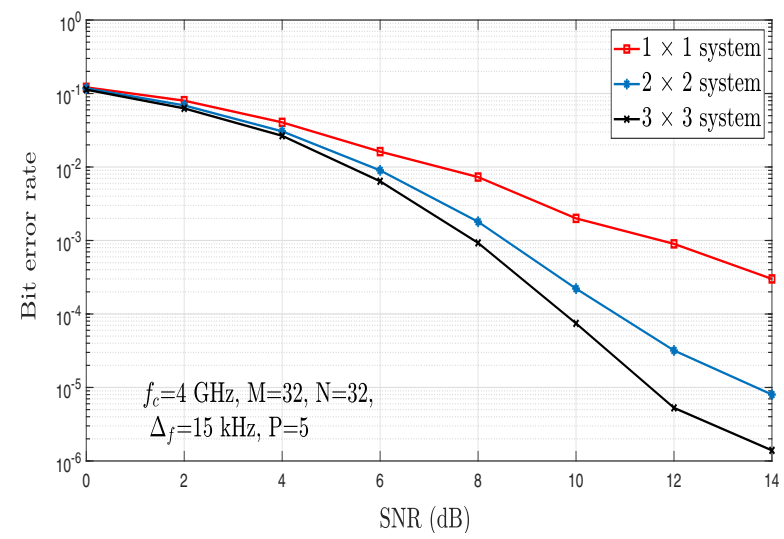

Fig. 2. BER performance of MIMO-OTFS for SISO, and $2 \times 2$ and $3 \times 3$ MIMO systems.

The maximum considered speed of $507.6 \mathrm{kmph}$ corresponds to $1880 \mathrm{~Hz}$ Doppler frequency at a carrier frequency of 4 GHz. Even at this high-Doppler value, MIMO-OTFS is found to achieve very good BER performance. We observe that, a BER of $10^{-5}$ is achieved at an SNR of about $14 \mathrm{~dB}$ for the $2 \times 2$ system, while the $S N R$ required to achieve the same BER reduces by about $2 \mathrm{~dB}$ for the $3 \times 3$ system. Thus, with the proposed detection algorithm, MIMO-OTFS brings in the advantages of linear increase in spectral efficiency with number of transmit antennas and the robustness of OTFS modulation in high-Doppler scenarios.

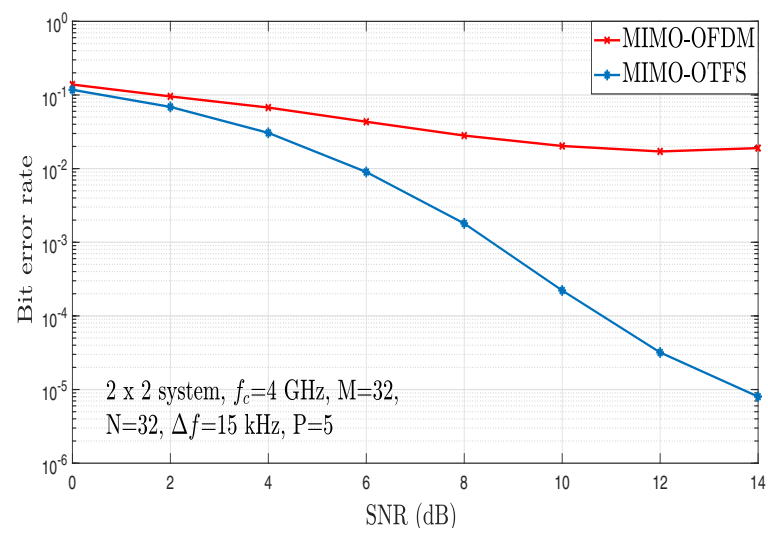

Fig. 3. BER performance comparison between MIMO-OTFS and MIMOOFDM in a $2 \times 2$ MIMO system.

Figure 3 shows the BER performance comparison between MIMO-OTFS and MIMO-OFDM in a $2 \times 2$ MIMO system. The maximum Doppler spread in the considered system is high $(1880 \mathrm{~Hz})$ which causes severe ICI in the TF domain. Because of the severe ICI, the performance of MIMO-OFDM is found to break down and floor at a BER value of about 
Transmitted OTFS symbols
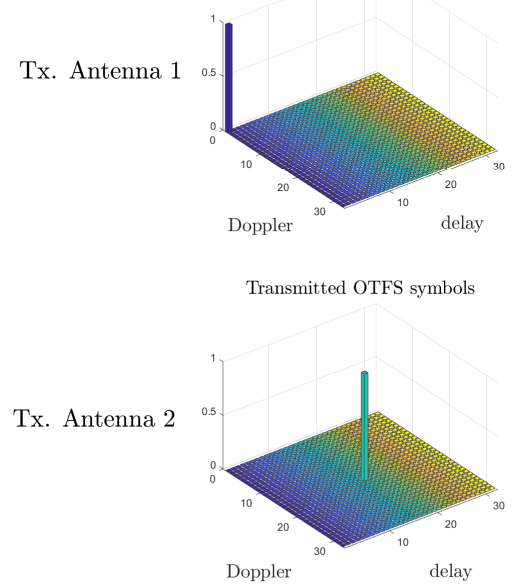

Channel
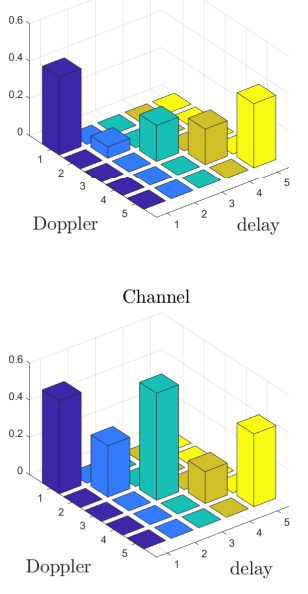

$2 \times 1$ system, $\mathrm{SNR}=4 \mathrm{~dB}$

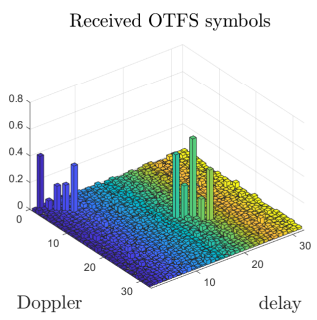

Fig. 4. Illustration of pilots and channel response in delay-Doppler domain in a $2 \times 1$ MIMO-OTFS system.

$2 \times 10^{-2}$. However, MIMO-OTFS is able to achieve a BER of $10^{-5}$ at an SNR value of about $14 \mathrm{~dB}$. This is because OTFS uses the delay-Doppler domain for signaling instead of TF domain. Thus, the BER plots clearly illustrate the robust performance of MIMO-OTFS and its superiority over MIMOOFDM under rapidly varying channel conditions.

\section{Channel Estimation For MiMO-OTFS}

In this section, we relax the assumption of perfect channel knowledge and present a channel estimation scheme in the delay-Doppler domain. The scheme uses impulses in the delayDoppler domain as pilots. Figure 4 gives an illustration of the pilots, channel response, and received signal in a $2 \times 1 \mathrm{MIMO}$ system with the delay-Doppler profile and system parameters given in Tables I and II. Each transmit and receive antenna pair sees a different channel having a finite support in the delay-Doppler domain. The support is determined by the delay and Doppler spread of the channel [8]. This fact can be used to estimate the channel for all the transmit-receive antenna pairs simultaneously using a single MIMO-OTFS frame as described below.

The OTFS input-output relation for $p$ th transmit antenna and $q$ th receive antenna pair can be written as

$\hat{x}_{q}[k, l]=\sum_{m=0}^{M-1} \sum_{n=0}^{N-1} x_{p}[n, m] \frac{1}{M N} h_{w_{q p}}\left(\frac{k-n}{N T}, \frac{l-m}{M \Delta f}\right)+v_{q}[k, l]$.

If we transmit

$$
\begin{aligned}
x_{p}[n, m] & =1 \text { if }(n, m)=\left(n_{p}, m_{p}\right) \\
& =0 \forall(n, m) \neq\left(n_{p}, m_{p}\right),
\end{aligned}
$$

as pilot from the $p$ th antenna, the received signal at the $q$ th antenna will be

$$
\hat{x}_{q}[k, l]=\frac{1}{M N} h_{w_{q p}}\left(\frac{k-n_{p}}{N T}, \frac{l-m_{p}}{M \Delta f}\right)+v_{q}[k, l] .
$$

We can estimate $\frac{1}{M N} h_{w_{q p}}\left(\frac{k}{N T}, \frac{l}{M \Delta f}\right)$ from (16), since, being the pilots, $n_{p}$ and $m_{p}$ are known at the receiver a priori.
From this, we can get the equivalent channel matrix $\hat{\mathbf{H}}_{q p}$ using the vectorized formulation. From (16) we also see that, due to the 2D-convolution input-output relation, the impulse at $(n, m)=\left(n_{p}, m_{p}\right)$ is spread by the channel only to the extent of the support of the channel in the delay-Doppler domain. Thus, if we send the pilot impulses from the transmit antennas with sufficient spacing in the delay-Doppler domain, they will be received without overlap. Hence, we can estimate the channel responses corresponding to all the transmit-receive antenna pairs simultaneously and get the estimate of the equivalent MIMO-OTFS channel matrix $\hat{\mathbf{H}}_{\text {MIмо }}$ using a single MIMOOTFS frame. This is illustrated in Fig. 4 for a $2 \times 1$ MIMOOTFS system with frame size $(M, N)=(32,32)$ at an SNR value of $4 \mathrm{~dB}$. The first antenna transmits the pilot impulse at $\left(n_{1}, m_{1}\right)=(0,0)$ and the second antenna transmits the pilot impulse at $\left(n_{2}, m_{2}\right)=(16,16)$ in the delay-Doppler domain. We observe that the impulse response $h_{w_{11}}\left(\frac{k-n_{1}}{N T}, \frac{l-m_{1}}{M \Delta f}\right)$ and $h_{w_{12}}\left(\frac{k-n_{2}}{N T}, \frac{l-m_{2}}{M \Delta f}\right)$ are non-overlapping at the receiver. Thus they can be estimated simultaneously using a single pilot MIMO-OTFS frame.

\section{A. Performance results and discussions}

In this subsection, we present the BER performance of the MIMO-OTFS system using the estimated channel. We use the MIMO-OTFS channel estimation scheme described above, for estimating the equivalent channel matrix $\hat{\mathbf{H}}_{\text {мIмо }}$ and use the message passing algorithm for detection. The delay-Doppler profile and the simulation parameters are as given in Table I and Table II, respectively.

In Fig. 5, we plot the Frobenius norm of the difference between the equivalent channel matrix $\left(\mathbf{H}_{\text {мімо }}\right)$ and the estimated equivalent channel matrix $\left(\hat{\mathbf{H}}_{\text {мімо }}\right.$ ) (a measure of estimation error) as a function of pilot SNR for a $2 \times 2$ MIMO-OTFS system with system parameters as in Tables I and II. We observe that, as expected, the Frobenius norm of the difference matrix decreases with pilot SNR. Figure 6 shows the corresponding BER performance using the proposed 


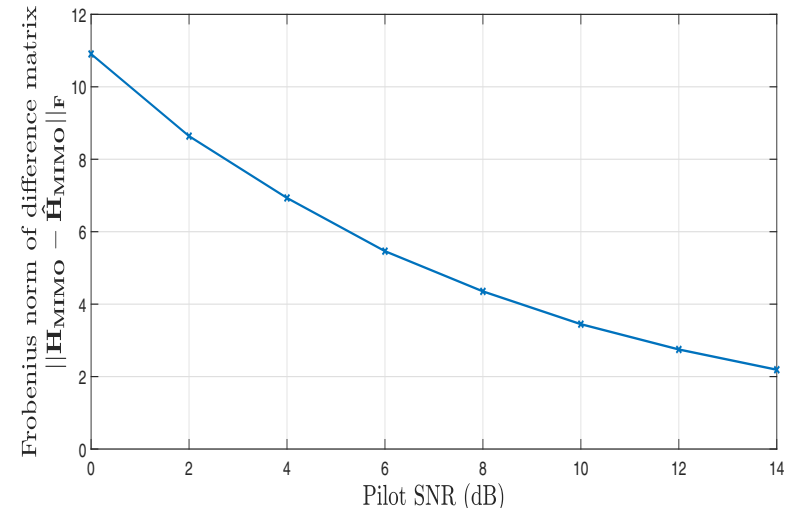

Fig. 5. Frobenius norm of the difference between the equivalent channel matrix ( $\left.\mathbf{H}_{\text {MIмо }}\right)$ and the estimated equivalent channel matrix $\left(\hat{\mathbf{H}}_{\text {MIмо }}\right)$ as a function of pilot SNR in a $2 \times 2$ MIMO-OTFS system.

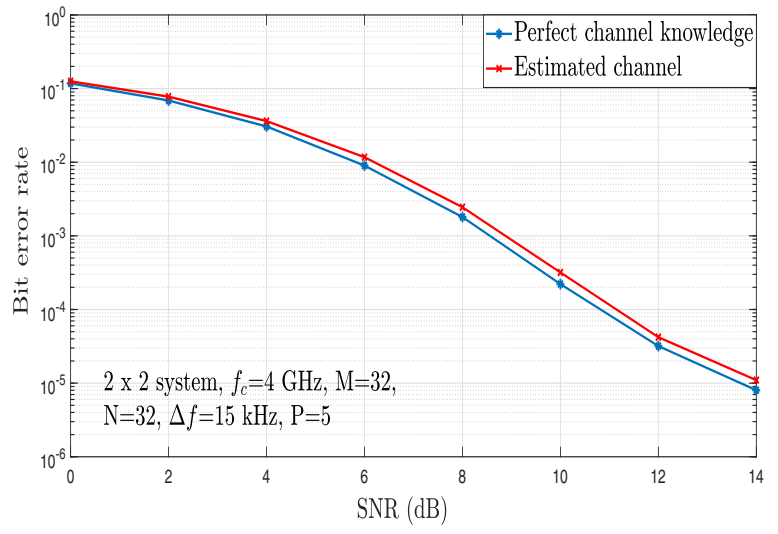

Fig. 6. BER performance of MIMO-OTFS system using the estimated channel in a $2 \times 2$ MIMO-OTFS system.

channel estimation scheme for the $2 \times 2$ MIMO-OTFS system. It is observed that the BER performance achieved with the estimated channel is quite close to the performance with perfect channel knowledge. For example, a BER of $2 \times 10^{-5}$ is achieved at SNR values of about $12.5 \mathrm{~dB}$ and $13 \mathrm{~dB}$ with perfect channel knowledge and estimated channel knowledge, respectively. At the considered maximum Doppler frequency of $1880 \mathrm{~Hz}$, channel estimation in the time-frequency domain leads to inaccurate estimation because of the rapid variations of the channel in time. On the other hand, the sparse channel representation in the delay-Doppler domain is time-invariant over a larger observation time. This, along with the OTFS channel-symbol coupling (2D periodic convolution) in the delay-Doppler domain, enables the proposed channel estimation for MIMO-OTFS to be simple and efficient.

\section{CONCLUSIONS}

We investigated signal detection and channel estimation aspects of MIMO-OTFS under high-Doppler channel conditions. We developed a vectorized formulation of the input-output relationship for MIMO-OTFS which enables MIMO-OTFS signal detection using a variety of detection algorithms. We presented a low complexity iterative algorithm for MIMOOTFS detection based on message passing. The algorithm was shown to achieve very good BER performance even at high Doppler frequencies (e.g., $1880 \mathrm{~Hz}$ ) in a $2 \times 2 \mathrm{MIMO}$ system where MIMO-OFDM was shown to floor in its BER performance. We also presented a channel estimation scheme in the delay-Doppler domain, where delay-Doppler impulses are used as pilots. The proposed channel estimation scheme was shown to be efficient and the BER degradation was small as compared to the performance with perfect channel knowledge. The sparse nature of the channel in the delay-Doppler domain which is time-invariant over a larger observation time enabled the proposed estimation scheme to be simple and efficient.

\section{REFERENCES}

[1] W. C. Jakes, Microwave Mobile Communications, New York: IEEE Press, reprinted, 1994.

[2] A. Goldsmith, Wireless Communications, Cambridge Univ. press, 2005.

[3] T. Wang, J. G. Proakis, E. Masry, and J. R. Zeidler, "Performance degradation of OFDM systems due to Doppler spreading," IEEE Trans. Wireless Commun., vol. 5, no. 6, pp. 1422-1432, Jun. 2006.

[4] T. Strohmer and S. Beaver, "Optimal OFDM design for time-frequency dispersive channels," IEEE Trans. Commun., vol. 51, no. 7, pp. 11111122, Jul. 2003.

[5] F-M. Han and X-D. Zhang, "Hexagonal multicarrier modulation: a robust transmission scheme for time-frequency dispersive channels," IEEE Trans. Signal Process., vol. 55, no. 5, pp. 1955-1961, May 2007.

[6] F-M. Han and X-D. Zhang, "Wireless multicarrier digital transmission via Weyl-Heisenberg frames over time-frequency dispersive channels," IEEE Trans. Commun., vol. 57, no. 6, pp. 1721-1733, Jun. 2009.

[7] R. Hadani and A. Monk, "OTFS: A new generation of modulation addressing the challenges of 5G," arXiv:1802.02623 [cs.IT] 7 Feb 2018

[8] A. Monk, R. Hadani, M. Tsatsanis, S. Rakib, "OTFS - orthogonal time frequency space: a novel modulation technique meeting $5 \mathrm{G}$ high mobility and massive MIMO challenges," arXiv:1608.02993 [cs.IT] 9 Aug 2016.

[9] R. Hadani, S. Rakib, M. Tsatsanis, A. Monk, A. J. Goldsmith, A. F. Molisch, and R. Calderbank, "Orthogonal time frequency space modulation," Proc. IEEE WCNC'2017, pp. 1-7, Mar. 2017.

[10] R. Hadani, S. Rakib, A. F. Molisch, C. Ibars, A. Monk, M. Tsatsanis, J. Delfeld, A. Goldsmith, and R. Calderbank, "Orthogonal time frequency space (OTFS) modulation for millimeter-wave communications systems," in Proc. IEEE MTT-S Intl. Microwave Symp., pp. 681-683, Jun. 2017.

[11] L. Li, H. Wei, Y. Huang, Y. Yao, W. Ling, G. Chen, P. Li, and Y. Cai, "A simple two-stage equalizer with simplified orthogonal time frequency space modulation over rapidly time-varying channels," arXiv:1709.02505v1 [cs.IT] 8 Sep 2017.

[12] P. Raviteja, K. T. Phan, Q. Jin, Y. Hong, and E. Viterbo, "Lowcomplexity iterative detection for orthogonal time frequency space modulation," arXiv:1709.09402v1 [cs.IT] 27 Sep 2017.

[13] M. K. Ramachandran and A. Chockalingam, "MIMO-OTFS in highDoppler fading channels: signal detection and channel estimation," arXiv:1805.02209 [cs.IT] 6 May 2018.

[14] A. R. Reyhani, A. Farhang, M. Ji, R-R. Chen, B. Farhang-Boroujeny, "Analysis of discrete-time MIMO OFDM-based orthogonal time frequency space modulation," arXiv:1710.07900v1 [cs.IT] 22 Oct 2017.

[15] T. Dean, M. Chowdhury, and A. Goldsmith, "A new modulation technique for Doppler compensation in frequency-dispersive channels," Proc. IEEE PIMRC'2017, Oct. 2017.

[16] A. Farhang, A. Rezazadeh Reyhani, L. E. Doyle, and B. FarhangBoroujeny, "Low complexity modem structure for OFDM-based orthogonal time frequency space modulation," IEEE Wireless Commun. Lett., doi: 10.1109/LWC.2017.2776942, Nov. 2017.

[17] K. R. Murali and A. Chockalingam, "On OTFS modulation for highDoppler fading channels," Proc. ITA'2018, San Diego, Feb. 2018.

[18] Y.G. Li, J.H. Winters, and N.R. Sollenberger, "MIMO-OFDM for wireless communications: signal detection with enhanced channel estimation," IEEE Trans. Commun., vol. 50, no. 9, pp. 1471-1477, Sep. 2002.

[19] F. Hlawatsch and G. Matz, Wireless Communications Over Rapidly Time-Varying Channels, Academic Press, 2011.

[20] A. Fish, S. Gurevich, R. Hadani, A. M. Sayeed, and O. Schwartz, "Delay-Doppler channel estimation in almost linear complexity," IEEE Trans. Inf. Theory, vol. 59, no. 11, pp. 7632-7644, Nov. 2013. 